Reprint from

F. Melchers et al. (Eds.)

Progress in Immunology, Vol. VII

C Springer-Verlag Berlin Heidelberg 1989

Printed in Germany. Not for Sale.

Reprint is only allowed with permission from Springer-Verlag.

Springer-Verlag Berlin Heidelberg New York London Paris Tokyo Hong Kong 


\title{
Apoptosis in Monoclonal Antibody-Induced Tumor Regression
}

\author{
P.H. Krammer, B.C. Trauth, V. Bier, J. Dhein, W. Falk, G. Garcin, C. Klas, \\ W. Müller, A. Oehm, A. Peters, S. Matzku, P. Möller, and K.-M. Debatin
}

\section{INTRODUCTION}

A variety of cell surface molecules are involved in growth control of normal and malignant cells and transmit lateral or vertical signals regulating cell replication. It is the purpose of our experiments to define such molecules and their function. Therefore, we raised monoclonal antibodies in mice against the malignant human B cell line SKW6.4 and tested them for growth inhibition of SKW6.4 cells in vitro. The following article describes the activity of a selected monoclonal antibody, anti-APO-1, which induces growth inhibition of SKW6.4 and a variety of other cells by induction of programmed cell death (apoptosis).

\section{RESULTS AND DISCUSSION}

After repeated injection of $1 \times 10^{7}$ SKW6.4 cells (once per week, four times) into Balb/c mice, spleen cells were used for fusion (Köhler and Milstein 1975) and the resulting hybridoma supernatants screened for growth inhibition of SKW6.4 cells in $200 \mu$ l cultures. Between 10 $15,000$ hybridomas were screened and one monoclonal antibody, named anti-APO-1 ( $\operatorname{IgG} 3, \kappa)$ was selected because it had the most drastic effect on growth of SKW6.4 cells. By Scatchard analysis we determined that the antibody recognized about 40,000 epitopes on SKW6.4 cells with high affinity $\left(\mathrm{KD}=1.9 \times 10^{-10}\right)$. Anti-APO-1 precipitated a protein antigen of a molecular weight of 52,000 which we called APO-1. We also saw coprecipitation of a minor $25 \mathrm{kD}$ band which is either a breakdown product of APO-1 or another noncovalently associated protein. Anti-APO-1 showed characteristic effects on SKW6.4 cells in culture and induced a process which morphologically appeared like programmed cell death, apoptosis. Apoptosis is the most common form of death in eukaryotic organisms (Duvall and Wyllie 1986). It is observed in embryogenesis and metamorphosis, whenever old tissue is replaced by new tissue, in tissue atrophy, and in tumor regression. Morphologically it is characterized by fragmentation of the nucleolus, segmentation of the nucleus, condensation of the cytoplasm, zeiosis (membrane blebbing), and biochemically by a fragmentation of genomic DNA into multimers of 180 bp by activation of an endonuclease which cuts the DNA between the nucleosomes (Wyllie 1980). We found apoptosis in various malignant $\mathrm{T}$ and $\mathrm{B}$ cell lines after incubation with anti-APO-1 for 1 - $24 \mathrm{hrs}$. Such cells show a reduced growth rate. In fact, $1-10 \mathrm{ng} / \mathrm{ml}$ of anti-APO-1 reduce growth of SKW6.4 cells and other malignant $T$ and $B$ cells in vitro by over $95 \%$. A variant of SKW6.4 cells selected by prolonged growth in vitro under antibody pressure with anti-APO-1 did not undergo apoptosis even at concentrations of anti-APO-1 which were about 25,000 times higher than used for the wildtype SKW6.4 cells.

We next wanted to investigate the signal requirements for anti-APO-1 and found that crosslinking of the APO-1 antigen is necessary for apoptosis to be induced. This is also supported by the fact that 4 - 5 times less anti-APO- 1 antibody is needed for induction of apoptosis when protein $A$ as an Fc-binding crosslinker is mixed to the culture. In addition, anti-APO-1 immobilized on beads was perfectly capable of inducing apoptosis. This indicates that anti-APO-1 is not internalized for induction of apoptosis. These data suggest that the APO-1 antigen when triggered by the anti-APO-1 antibody delivers a signal which leads to the induction of the endogenous suicide process. 
We also investigated the representation of the APO-1 antigen on normal cells and found that APO-1 is absent from the majority of resting $\mathrm{T}$ and $\mathrm{B}$ lymphocytes. However, after activation of T lymphocytes with PMANPHA and Interleukin-2 the APO-1 antigen appears on the cell surface after about one day of activation. Only a slight additional increase of expression of APO-1 on the cell surface is observed on day 6 of activation. Day 1- and day 6-activated T cells, however, show a considerable difference in sensitivity for induction of apoptosis by antiAPO-1. Whereas hardly any apoptosis occurs on day 1-activated T cells, apoptosis is induced on day 6-activated T cells. Likewise, the majority of resting B cells is negative for APO-1 expression. B cells activated with pokeweed mitogen, however, express the APO-1 antigen and after incubation with the anti-APO-1 antibody cease to secrete IgM, probably due to induction of apoptosis. Apart from activated lymphocytes, the APO-1 antigen is expressed in a restricted fashion on a variety of normal tissues. Thus, as an example APO-1 is found in a basolateral distribition on epithelial cells of the colon mucosa. Interestingly, a number of colon carcinomas have lost APO-1 and others show a strong expression of APO-1.

Apart from the above cells, we tested a number of in vitro cell lines for the expression of APO1 and the induction of apoptosis by anti-APO-1. We found that the human $B$ cell lines SKW6.4, CESS, and BJAB were positive as well as the human $\mathrm{T}$ cell lines Jurkat, Molt, and CCRF-CEM. The human myeloid cell line U937, the gibbon T cell line MLA-144 and the mouse $T$ cell line EL 4 were negative. The cell lines of the above panel which express APO-1 also underwent apoptosis after incubation with anti-APO-1. The effects of anti-APO-1 in vitro prompted us to test whether the antibody was also effective in vivo. Although $B J A B$ was the least anti-APO-1 sensitive of our in vitro B cell lines we selected this cell line for our in vivo experiments because it was the only line that grew to a large tumor in nu/nu mice. BJAB carries about 15,000 APO-1 antigens on the cell.surface. Approximately five weeks after injection of $5 \times 10^{7} \mathrm{BJAB}$ cells into nu/nu mice $\mathrm{BJAB}$ tumors had reached a diameter of about $1-2.5 \mathrm{~cm}$. The tumor carrying animals were then injected once intravenously with $500 \mu \mathrm{g}$ of anti-APO- 1 or a similar amount of isotype matched binding or non-binding control antibody, respectively. Experiments using radiolabelled antibodies indicated that anti-APO-1 and binding control antibodies localized in the tumor tissue while non-binding antibodies only showed background localization. Control antibodies did not have any effect on the BJAB tumors in the $\mathrm{nu} / \mathrm{nu}$ mice. However, anti-APO-1 led to complete tumor regression in most animals between 5 and 14 days after injection of the antibody. Histological thin sections of tumors before complete regression showed that anti-APO-1 had also induced apoptosis in vivo.

In conclusion, therefore, our experiments showed that the APO-1 antigen is associated with growth and differentiation of human lymphocytes. The anti-APO-1 antibody induces growth inhibition and programmed cell death (apoptosis) of activated normal human lymphocytes and $\mathrm{B}$ and $\mathrm{T}$ cell tumors. Further experiments indicated that these processes can also be induced in a number of non-lymphoid tumors. The effect of anti-APO-1 is complement independent, and a transduction of the APO- 1 mediated trans-membrane signal depends on efficient APO-1 crosslinking. Finally, a single intravenous injection of anti-APO-1 induces a rapid regression of a large tumor mass of the human Burkitt-like $B$ cell lymphoma BJAB in nu/nu mice.

Future experiments are aimed at defining the physiological role of APO-1 and establishing a molecular understanding of growth inhibition and the mechanism of apoptosis. Furthermore, it is apparent that anti-APO-1 might be used as a diagnostic and putative therapeutic tool in oncology.

\section{REFERENCES:}

Duvall E, Wyllie AH (1986) Death and the cell. Immunology today 7:115

Köhler G, Milstein C (1975) Continuous cultures of fused cells secreting antibody of predefined specificity. Nature 256:495

Wyllie AH (1980) Glucocorticoid-induced thymocyte apoptosis is associated with endogenous endonuclease activation. Nature 284:555. 